\title{
ÍNDICES DE REATIVIDADE QUÍMICA A PARTIR DA TEORIA DO FUNCIONAL DE DENSIDADE: FORMALISMO E PERSPECTIVAS
}

Hélio Anderson Duarte*

Departamento de Química - ICEx, Universidade Federal de Minas Gerais, Av. Antônio Carlos, 6627, Pampulha, 31270-901 Belo Horizonte - MG

Recebido em 30/5/00; aceito em 6/11/00

\begin{abstract}
CHEMICAL REACTIVITY INDEXES FROM DENSITY FUNCTIONAL THEORY: FORMALISM AND PERSPECTIVES. The fundaments of the modern Density Functional Theory (DFT), its basic theorems, principles and methodology are presented. This review also discuss important and widely used concepts in chemistry but that had not been precisely defined until the development of the DFT. These concepts were proposed and used from an empirical base, but now their precise definition are well established in the DFT formalism. Concepts such as chemical potential (electronegativity), hardness, softness and Fukui function are presented and their consequences to the understanding of chemical reactivity are discussed.
\end{abstract}

Keywords: density functional theory; reactivity indexes; hardness; softness.

\section{INTRODUÇÃO}

A Teoria de Drude ${ }^{1}$ de metais foi provavelmente a primeira tentativa de se usar a densidade eletrônica como variável básica na descrição de um sistema eletrônico. Isto aconteceu apenas três anos depois da descoberta do elétron por Thompson em 1897. Drude aplicou a teoria cinética dos gases a um metal, considerado como um gás homogêneo de elétrons, para desenvolver a sua teoria sob condução térmica e elétrica. Da mesma forma que na teoria cinética dos gases, as interações elétron-elétron e elétron-íon eram desprezadas. Mais tarde, Sommerfeld usou a distribuição quântica de Fermi-Dirac no lugar da distribuição clássica de Maxwell-Boltzman para a distribuição da velocidade eletrônica no modelo de Drude. O modelo de Drude-Sommerfeld continha várias simplificações e, consequentemente, não foi possível fazer uma descrição precisa de um sólido a partir deste modelo, embora ele tenha sido considerado como um grande avanço naquela época.

$\mathrm{O}$ próximo passo foi o modelo de Thomas-Fermi ${ }^{2}$, proporcionando uma sensível melhora em relação ao modelo de Drude-Sommerfeld. Neste modelo, partiu-se do pressuposto de que os elétrons estão distribuídos uniformemente no espaço. Usando argumentos estatísticos para aproximar a distribuição dos elétrons, Thomas e Fermi desenvolveram o funcional de energia de Thomas-Fermi (TF) (Eq. 1 com $\mathrm{Cx}=0$ ). $\mathrm{O}$ modelo de Thomas-Fermi-Dirac (TFD) inclui, em adição, a energia de troca para um gás de elétrons desenvolvido por Dirac ${ }^{3}$. O funcional de energia (E) de TFD é dado por

$$
\begin{aligned}
& \mathrm{E}_{\mathrm{TFD}}[\rho]=\mathrm{C}_{\mathrm{F}} \int \rho(\mathrm{r})^{5 / 3} \mathrm{dr}+\int \rho(\mathrm{r}) v(\mathrm{r}) \mathrm{dr}+\frac{1}{2} \iint \frac{\rho\left(\mathrm{r}_{1}\right) \rho\left(\mathrm{r}_{2}\right)}{\left|\mathrm{r}_{1}-\mathrm{r}_{2}\right|} \mathrm{dr}-\mathrm{C}_{\mathrm{x}} \int \rho(\mathrm{r})^{4 / 3} \mathrm{dr} \\
& \text { onde } \mathrm{C}_{\mathrm{F}}=\frac{3}{10}\left(3 \pi^{2}\right)^{2 / 3} \text { e } \mathrm{C}_{\mathrm{x}}=\frac{3}{4}\left(\frac{3}{\pi}\right)^{1 / 3} .
\end{aligned}
$$

Os quatro termos da direita da Eq. (1) correspondem à energia cinética, ao potencial externo ${ }^{i}$, ao potencial de Coulomb e à energia de troca, respectivamente. $\rho$ e $r$ correspondem a densidade eletrônica e as coordenadas, respectivamente.

\footnotetext{
e-mail: duarteh@netuno.qui.ufmg.br

Potencial externo normalmente consiste na posição e na carga dos núcleos dos átomos que formam a molécula.
}

O modelo TFD pode ser resolvido apenas numericamente e a solução da equação variacional

$$
\delta\left\{\mathrm{E}_{\mathrm{TDF}}[\rho]-\mu \mathrm{N}(\rho)\right\}=0
$$

onde $\mu$ é o potencial químico e $\mathrm{N}$ o número de partículas, é essencialmente independente do número de partículas. Porém, estes modelos de funcionais clássicos (TF e TFD) não podem competir em precisão com métodos auto-consistentes como, por exemplo, o método de Hartree-Fock. O modelo de TFD falha ao reproduzir a estrutura quântica de camadas dos átomos. Além disso, o método não prediz ligação molecular e os resultados para átomos não são muito precisos. A TFD é supersimplificada e não permite predições quantitativas ${ }^{4,5}$.

Foi em 1964, com a publicação de dois teoremas por Hohenberg e Kohn, que o uso da densidade eletrônica como variável básica foi rigorosamente legitimado. Eles forneceram os fundamentos da teoria do funcional de densidade moderna (DFT, do Inglês Density Functional Theory) e mostraram que os modelos baseados no funcional de energia TFD devem ser vistos como uma aproximação de uma teoria exata. Os dois teoremas ${ }^{6}$ mostram que existe um funcional de energia exato da densidade eletrônica $E[\rho]$ e um princípio variacional exato para este funcional, semelhante a Eq. (2). E, em 1965, Kohn e Sham ${ }^{7}$ propuseram uma forma de contornar o problema de se encontrar o funcional de energia exato (o método KS). Desde a publicação destes dois artigos, a DFT tem atraído cada vez mais a atenção da comunidade científica e, atualmente, ela é largamente usada para se estudar sistemas cada vez mais complexos.

Interessante e cuidadosa comparação entre os métodos computacionais usados para fazer cálculos ab initio convencionais e DFT é apresentada no artigo de Morgon e Custódio ${ }^{8}$ publicado neste periódico. O desenvolvimento da metodologia computacional para fazer cálculos DFT leva, invariavelmente, as equações matemáticas semelhantes às equações do método de Hartree-Fock-Roothaan. Porém, apesar da semelhança, os resultados de cálculos DFT devem ser interpretados à luz da Teoria do Funcional de Densidade. Diferentemente de outros métodos, tais como métodos semi-empíricos, que tentam se aproximar do método Hartre-Fock tanto quanto possível, a DFT está relacionada com a solução exata do problema de muitos elétrons.

Esta revisão apresenta os fundamentos da teoria moderna do funcional de densidade, seus teoremas básicos, princípios e 
metodologia. Conceitos importantes na descrição teórica de reações químicas, tais como potencial químico, conceitos de maciez e dureza (hardness e softness) e outros índices de reatividade química redefinidos, de uma maneira exata a partir da Teoria do Funcional de Densidade são discutidos.

\section{TEOREMAS DE HOHEMBERG-KOHN}

\section{Primeiro Teorema}

No artigo de 1964, Hohenberg e Kohn ${ }^{6}$ (HK) consideraram um sistema de N-elétrons descrito pelo Hamiltoniano não relativístico:

$$
\hat{\mathrm{H}}=\hat{\mathrm{T}}+\hat{\mathrm{V}}_{\mathrm{e}}+\hat{\mathrm{U}}
$$

onde $\hat{\mathrm{T}}$ é a energia cinética, $\hat{\mathrm{V}}_{\mathrm{e}} \mathrm{o}$ operador de repulsão elétron-elétron o qual inclui a repulsão Coulombiana e todos os termos não clássicos (troca e correlação) e Û é o potencial externo com respeito aos elétrons, normalmente devido às cargas dos núcleos $Z \alpha$ :

$$
\hat{\mathrm{U}}=\sum_{\mathrm{i}}^{\mathrm{N}} \sum_{\alpha}-\frac{\mathrm{Z}_{\alpha}}{\left|\mathrm{R}_{\alpha}-\mathrm{r}_{\mathrm{i}}\right|}=\sum_{\mathrm{i}} \mathrm{v}\left(\mathrm{r}_{\mathrm{i}}\right)
$$

A densidade eletrônica é definida pela equação seguinte:

$$
\rho\left(\mathrm{r}_{1}\right)=\int \ldots \int \Psi\left(\mathrm{r}_{1}, \mathrm{r}_{2}, \ldots, \mathrm{r}_{\mathrm{n}}\right)^{*} \Psi\left(\mathrm{r}_{1}, \mathrm{r}_{2}, \ldots, \mathrm{r}_{\mathrm{n}}\right) \mathrm{dr}_{2}, \mathrm{dr}_{3} \ldots \mathrm{dr}_{\mathrm{n}}
$$

onde $\Psi\left(\mathrm{r}_{1}, \mathrm{r}_{2}, \ldots \mathrm{r}_{\mathrm{n}}\right)$ é a solução do estado fundamental do Hamiltoniano.

Originalmente, supos-se que o estado fundamental não é degenerado. A energia total do sistema é então dada por

$$
\mathrm{E}_{\mathrm{o}}=\int \Psi^{*} \hat{\mathrm{H}} \Psi \mathrm{dr}_{1} \mathrm{dr}_{2} \ldots \mathrm{dr}_{\mathrm{n}}=\langle\Psi|\hat{\mathrm{H}}| \Psi\rangle
$$

ou, se o potencial externo é separado em um funcional trivial da densidade eletrônica, por

$$
\mathrm{E}_{\mathrm{o}}=\left\langle\Psi\left|\hat{\mathrm{T}}+\hat{\mathrm{V}}_{\mathrm{e}}\right| \Psi\right\rangle+\int \rho(\mathrm{r}) \mathrm{v}(\mathrm{r}) \mathrm{dr}
$$

$\hat{\mathrm{T}} \mathrm{e} \hat{\mathrm{V}}_{\mathrm{e}}$ aplica-se universalmente à todos os sistemas eletrônicos. O número de elétrons, $\mathrm{N}$, e o potencial externo, $\hat{\mathrm{U}}$, no qual os elétrons se movem, definem completamente o sistema de muitos elétrons. O primeiro teorema de HK estabelece que o potencial externo, $\hat{\mathrm{U}}$, é um funcional único de $\mathrm{\rho}(\mathrm{r})$, além de uma constante aditiva.

\section{Segundo Teorema}

$\mathrm{O}$ segundo teorema estabelece que qualquer aproximação da densidade eletrônica, $\tilde{\rho}(\mathrm{r})$, de tal modo que $\tilde{\rho}(\mathrm{r}) \geq 0$ e $\int \tilde{\rho}(\mathrm{r}) \mathrm{dr}=\mathrm{N}$, a energia total $\mathrm{E}[\tilde{\rho}] \geq \mathrm{E}[\rho]=\mathrm{E}_{\mathrm{o}}$.

A forma do operador de energia cinética e do operador da repulsão eletrônica é a mesma para qualquer sistema eletrônico com um dado número de partículas e potencial externo. Podese, então, definir um funcional universal,

$$
\mathrm{F}[\rho]=\left\langle\Psi\left|\hat{\mathrm{T}}+\hat{\mathrm{V}}_{\mathrm{e}}\right| \Psi\right\rangle
$$

No entanto, é preciso observar que $\tilde{\rho}(\mathrm{r})$ define seu próprio $\tilde{v}(\mathrm{r})$ e, consequentemente, o Hamiltoniano $\tilde{\mathrm{H}}$ e $\widetilde{\Psi}\left(\mathrm{r}_{1}, \mathrm{r}_{2}, \ldots, \mathrm{r}_{\mathrm{n}}\right)$. A função $\tilde{\Psi}\left(\mathrm{r}_{1}, \mathrm{r}_{2}, \ldots, \mathrm{r}_{\mathrm{n}}\right)$, por sua vez, pode ser usada como uma função tentativa para o sistema com o potencial externo $v(r)$. De acordo com o princípio variacional, temos

$E_{o}=E_{v}[\rho]=F[\rho]+\int v(r) \rho(r) d r \leq E_{v}[\tilde{\rho}]+\int v(r) \tilde{\rho}(r) d r$

$\mathrm{E}_{\mathrm{v}}$ se refere ao funcional da energia total com relação ao potencial externo $v(\mathrm{r})$.

Os dois teoremas de HK mostram como se pode determinar o estado fundamental de um sistema, com um dado potencial externo, usando a densidade eletrônica tridimensional como variável básica ao invés de usar a função de onda de N-elétrons, que é muito mais complexa.

$\mathrm{Na}$ prática, nós queremos usar o princípio variacional para encontrar $\rho(\mathrm{r})$ em um dado potencial externo $v(\mathrm{r})$, e não o oposto. O primeiro teorema de $\mathrm{HK}$ estabelece que se $\rho(\mathrm{r})$ é conhecido, pode-se determinar $v(r)$. Porém, dado uma densidade eletrônica tentativa, $\tilde{\rho}(\mathrm{r})$, como podemos garantir que $\tilde{\rho}(\mathrm{r})$ é "v-representável", ou seja, que corresponda à um potencial fisicamente realizável? Uma outra questão surge em relação a "N-representabilidade" da densidade eletrônica. A densidade eletrônica $\tilde{\rho}(\mathrm{r})$ é "N-representável" se ela pode ser obtida a partir de uma função de onda antissimétrica. Gilbert ${ }^{9}$ mostrou que qualquer densidade eletrônica razoável é "Nrepresentável", se as seguintes condições forem satisfeitas:

$$
\begin{aligned}
& \rho(\mathrm{r})>0 \\
& \int \rho(\mathrm{r}) \mathrm{dr}=\mathrm{N} \\
& \int\left|\nabla \rho(\mathrm{r})^{1 / 2}\right|^{2} \mathrm{dr}<\infty
\end{aligned}
$$

Felizmente, a Teoria do Funcional de Densidade pode ser formulada de tal forma que a condição da " $v$-representabilidade" pode ser contornada através da formulação de Levy ${ }^{10}$ da procura restrita (Levy Constrained Search). Seguindo Parr e Yang ${ }^{4}$,

$\mathrm{F}[\rho]=\left\langle\Psi_{\mathrm{o}}\left|\hat{\mathrm{T}}+\hat{\mathrm{V}}_{\mathrm{e}}\right| \Psi_{\mathrm{o}}\right\rangle=\min _{\Psi \rightarrow \rho}\left\langle\Psi\left|\hat{\mathrm{T}}+\hat{\mathrm{V}}_{\mathrm{e}}\right| \Psi\right\rangle$

ou seja, a procura restrita consiste em testar todas as densidades tentativas, e para cada densidade encontrar as funções de onda $\Psi\left(r_{1}, r_{2}, \ldots r_{n}\right)$ que minimizem $F[\rho]$ e que gerem aquela densidade. Substituindo a Eq. (11) na Eq. (9) temos

$\mathrm{E}_{0}=\min _{\rho}\left\{\min _{\Psi \rightarrow \rho}\left\langle\Psi\left|\hat{\mathrm{T}}+\hat{\mathrm{V}}_{\mathrm{e}}\right| \Psi\right\rangle+\int \rho(\mathrm{r}) \mathrm{v}(\mathrm{r}) \mathrm{dr}\right\}=\min _{\rho} \mathrm{E}_{\mathrm{v}}[\rho]$

A minimização interna está restrita a todas as funções de onda, $\Psi$, que fornece $r$, enquanto a minimização externa é feita sobre todos os possíveis $\rho$.

A formulação de Levy também elimina a limitação dos teoremas de HK, segundo os quais, o estado fundamental tem que ser não degenerado. Além do mais, nenhuma referência a " $v$-representabilidade" da densidade eletrônica é necessária, uma vez que $\rho(\mathrm{r})$ é originada a partir de uma função de onda antissimétrica. A questão da "N-representabilidade" da densidade eletrônica é uma condição que tem que ser satisfeita, porém é uma condição mais fácil de ser atendida como foi demonstrada por Gilbert ${ }^{9}$, qualquer densidade eletrônica razoável é N-representável se as Eqs (10a), (10b) e (10c) forem satisfeitas.

\section{EQUAÇÕES DE KOHN-SHAM}

De acordo com os teoremas de HK, o funcional energia é escrito como segue: 


$$
E_{v}[\rho]=F[\rho]+\int v(r) \rho(r) d r
$$

O estado fundamental satisfaz o princípio estacionário como requerido pelo princípio variacional (Eq. (9)),

$$
\delta\left\{\mathrm{E}_{v}[\rho]-\mu\left[\int \rho(\mathrm{r}) \mathrm{dr}-\mathrm{N}\right]\right\}=0
$$

O multiplicador de Lagrange, $\mu$, é o potencial químico dos elétrons e será discutido mais tarde. A função universal $F(\rho)$ é determinada pelo procedimento de minimização da Eq. (11).

Uma importante idéia introduzida por Kohn e Sham ${ }^{7}$ é usar o conceito de um sistema de referência de partículas independentes, ou seja, que não interagem. Eles rescreveram a Eq. (13) tornando explícita a repulsão elétron-elétron de Coulomb, e definindo uma nova função universal $G(\rho)$,

$$
E_{v}[\rho]=G[\rho]+\frac{1}{2} \iint \frac{\rho(r) \rho\left(r^{\prime}\right)}{\left|r-r^{\prime}\right|} d r d r^{\prime}+\int v(r) \rho(r) d r
$$

onde,

$$
G[\rho]=T_{s}[\rho]+E_{x c}[\rho]
$$

e $\mathrm{T}_{\mathrm{s}}[\rho]$ é o funcional de energia cinética de um sistema de elétrons que não interagem tendo a mesma densidade eletrônica do sistema que interage. $\mathrm{E}_{\mathrm{xc}}$ inclui o termo de interação elétron-elétron não clássica (troça e correlação) mais a parte residual da energia cinética, $\mathrm{T}[\rho]-\mathrm{T}_{\mathrm{s}}[\rho]$, onde $\mathrm{T}[\rho]$ é a energia cinética exata para o sistema de elétrons que interagem.

Torna-se importante ressaltar que as diferenças entre as definições da correlação eletrônica tradicionais (métodos ab initio convencional) e da DFT são geralmente ignoradas e funcionais de correlação são usualmente julgados pelo seu desempenho em reproduzir a energia de correlação tradicional, o qual é geralmente o único conhecido com um razoável grau de precisão ${ }^{11}$. Embora o formalismo DFT seja exato, a expressão para o funcional de troca-correlação não é conhecido em sua forma exata.

Orbital de um elétron pode, então, ser utilizado permitindo calcular precisamente a energia cinética via um procedimento auto-consistente:

$$
\mathrm{T}_{\mathrm{s}}[\rho]=\sum_{\mathrm{i}}^{\mathrm{N}}\left\langle\psi_{\mathrm{i}}\left|-\frac{1}{2} \nabla^{2}\right| \psi_{\mathrm{i}}\right\rangle
$$

Os orbitais são auto-funções do Hamiltoniano KS efetivo de um elétron,

$$
\mathrm{H}_{\mathrm{KS}}=-\frac{1}{2} \nabla^{2}+\mathrm{v}_{\text {eff }}(\mathrm{r})
$$

onde, o potencial efetivo, é definido como segue,

$$
v_{\text {eff }}(r)=v(r)+\int \frac{\rho\left(r^{\prime}\right)}{\left|r-r^{\prime}\right|} d r^{\prime}+v_{x c}(r)
$$

onde,

$$
v_{x c}(r)=\frac{\delta \mathrm{E}_{\mathrm{xc}}[\rho]}{\delta \rho(\mathrm{r})}
$$

A densidade eletrônica, $\rho(\mathrm{r})$, é dada por

$$
\rho(r)=\sum_{i}^{N}\left|\psi_{i}(r)\right|^{2}
$$

Portanto, os orbitais Kohn-Sham (KS), $\psi_{\text {i }}$, são obtidos a partir da equação de Schrödinger de um elétron

$$
\left(\frac{1}{2} \nabla^{2}+v_{\text {eff }}\right) \psi_{\mathrm{i}}=\varepsilon_{\mathrm{i}} \psi_{\mathrm{i}}
$$

As Equações (19), (20) e (21) representam o esquema KohnSham auto-consistente (KS-SCF). Uma vez que o potencial efetivo, $v_{\text {eff }}(r)$ depende da densidade eletrônica, $\rho(r)$, as equações de Kohn-Sham são resolvidas através de um procedimento auto-consistente.

No esquema Kohn-Sham, a densidade exata do estado fundamental de um sistema de elétrons que interagem é gerada a partir da solução de um problema auxiliar do sistema de elétrons que não-interagem definido de forma a fornecer a mesma densidade do estado fundamental. Este aspecto do método KS é discutido em detalhe nas referências 4 e 5 . As equações $\mathrm{KS}$, de maneira semelhante às equações de Hartree-Fock, fornecem equações de um elétron que descrevem sistemas de muitos elétrons. Além disso, as equações Kohn-Sham, em princípio, são exatas uma vez que incorporam totalmente os efeitos da correlação eletrônica (troca e correlação) e solução dessas equações formalmente equivalem a resolver exatamente o problema variacional da DFT. Em outras palavras, as equações KS vão além da aproximação de Hartree-Fock e ainda, oferecem uma grande vantagem: o potencial de troca-correlação é local. Na prática, isto significa que as integrais de quatro centros do método de Hartree-Fock usadas para calcular o termo de troca (que é um operador não local) não precisam ser realizadas, reduzindo-se desta forma o custo computacional.

A energia total é determinada usando a Eq. (15) ou pela fórmula

$$
\mathrm{E}=\sum \varepsilon_{\mathrm{i}}-\frac{1}{2} \int \frac{\rho(\mathrm{r}) \rho\left(\mathrm{r}^{\prime}\right)}{\left|\mathrm{r}-\mathrm{r}^{\prime}\right|} \mathrm{drdr}+\mathrm{E}_{\mathrm{xc}}[\rho]-\int v_{\mathrm{xc}}(\mathrm{r}) \rho(\mathrm{r}) \mathrm{dr}
$$

$\mathrm{O}$ esquema KS permite calcular a densidade eletrônica do estado fundamental. Todas as outras propriedades do sistema podem ser calculadas desde que os funcionais da densidade eletrônica apropriados sejam conhecidos.

A parte mais difícil para resolver as equações KS é determinar o funcional de troca-correlação $(X C) v_{x c}(r)$, uma vez que a exata forma analítica do potencial de troca-correlação não é conhecida. Muitos trabalhos têm sido devotados ao desenvolvimento e teste das diferentes aproximações dos funcionais de troca-correlação $^{12,13}$. A aproximação da densidade local baseada no modelo de gás homogêneo de elétrons ${ }^{14,15}$, a aproximação do gradiente generalizado ${ }^{16-21}$, o funcional LAP (Laplaciano), que usa a densidade de energia cinética e o Laplaciano da densidade eletrônica, são alguns dos funcionais de XC mais usados no cálculo de estrutura eletrônica. Os funcionais híbridos que incluem parte da energia de troca exata (termo de troca do método de Hartree-Fock) têm sido largamente utilizados principalmente no estudo de sistemas orgânicos. No entanto, vale ressaltar que estes funcionais não são puros uma vez que o potencial efetivo $v_{\text {eff }}(r)$ deixa de ser um operador local.

\section{O SIGNIFICADO DAS ENERGIAS DOS ORBITAIS KS E A EXTENSÃO DA DFT AO NÚMERO DE OCUPAÇÃO NÃO-INTEIRO}

O teorema de $\mathrm{Janak}^{22}$ e a conseqüente extensão da DFT ao número de ocupação não-inteiro é apresentada, seguindo a formulação de Mermin $^{23}$ e Perdew et al. ${ }^{24}$.

Primeiramente, a definição de $\mathrm{T}_{\mathrm{s}}(\rho)$ é generalizada, onde todos os possíveis números de ocupação $n_{i}$ e os orbitais ortonormais $\psi_{\mathrm{i}}$ são procurados: 
$\mathrm{T}_{\mathrm{J}}[\rho]=\min _{\left\{\mathrm{n}_{\mathrm{i}}, \psi_{\mathrm{i}}\right\} \rightarrow \rho}\left(\sum_{\mathrm{i}} \mathrm{n}_{\mathrm{i}}\left\langle\psi_{\mathrm{i}}\left|-\frac{1}{2} \nabla^{2}\right| \psi_{\mathrm{i}}\right\rangle\right)$

o subscrito $\mathrm{J}$ indica Janak. A densidade $\rho(\mathrm{r})$ é, agora, definido como segue

$$
\rho(\mathrm{r})=\sum_{\mathrm{i}} \mathrm{n}_{\mathrm{i}}\left|\psi_{\mathrm{i}}(\mathrm{r})\right|^{2}
$$

Portanto, é possível expressar a energia total em termos de $n_{\mathrm{i}}$ e $\psi_{\mathrm{i}}$,

$$
\begin{aligned}
& E[\rho]=T_{J}[\rho]+E_{x c}[\rho]+J[\rho]+\int v(r) \rho(r) d r \\
& =\sum_{i} n_{i}\left\langle\psi_{i}\left|-\frac{1}{2} \nabla^{2}\right| \psi_{i}\right\rangle+E_{x c}[\rho]+J[\rho]+\int v(r) \rho(r) d r
\end{aligned}
$$

com

$$
J[\rho]=\frac{1}{2} \iint \frac{\rho(\mathrm{r}) \rho\left(\mathrm{r}^{\prime}\right)}{\left|\mathrm{r}-\mathrm{r}^{\prime}\right|} \mathrm{drdr}
$$

Seguindo Janak ${ }^{22}$, usando a regra da cadeia para derivadas de funcional, a expressão da energia total pode ser diferenciada com respeito ao número de ocupação $\mathrm{n}_{\mathrm{i}}$ para fornecer,

$$
\begin{aligned}
& \frac{\partial \mathrm{E}}{\partial \mathrm{n}_{\mathrm{i}}}=\left\langle\psi_{\mathrm{i}}\left|-\frac{1}{2} \nabla^{2}\right| \psi_{\mathrm{i}}\right\rangle+\int \frac{\delta}{\delta \rho(\mathrm{r})}\left\{\mathrm{f}[\rho]+\mathrm{E}_{\mathrm{xc}}[\rho]+\int \mathrm{v}(\mathrm{r}) \rho(\mathrm{r}) \mathrm{dr} r \frac{\partial \rho(\mathrm{r})}{\partial \mathrm{n}_{\mathrm{i}}} \mathrm{dr}\right. \\
& =\left\langle\psi_{\mathrm{i}}\left|-\frac{1}{2} \nabla^{2}\right| \psi_{\mathrm{i}}\right\rangle+\int v_{\text {eff }}(\mathrm{r}) \psi_{\mathrm{i}}(\mathrm{r}) \psi_{\mathrm{i}}(\mathrm{r}) \mathrm{dr} \\
& =\varepsilon_{\mathrm{i}}
\end{aligned}
$$

Esta equação estabelece uma regra aufbau, o que significa que o funcional de energia, Eq. (26), é minimizado quando se coloca $n_{i}=1$ para os $\mathrm{N}$ orbitais $\psi_{i}$ mais baixos em energia e $\mathrm{n}_{\mathrm{i}}=0$ para os outros orbitais. Ocupação fracionária dos orbitais é possível apenas dentro de um conjunto de orbitais degenerados ao nível de Fermi. Porém, para sistemas de elétrons que não interagem entre si e que são n-representáveis, esta regra não se aplica ${ }^{25}$. A Eq. 28 também se aplica para sistemas com número não inteiro de elétrons, ou seja, número fracionário $\left(\mathrm{N}=\sum_{\mathrm{i}} \mathrm{n}_{\mathrm{i}}\right)$. De acordo com Perdew et al. ${ }^{24}$, a Eq. (28) fornece um significado físico do orbital KS ocupado de maior energia, $\varepsilon_{\max }$, ou seja,

$\varepsilon_{\max }=\left\{\begin{array}{l}-\mathrm{I}\left(\mathrm{N}_{\mathrm{Z}}-1<\mathrm{N}<\mathrm{N}_{\mathrm{Z}}\right) \\ -\mathrm{A}\left(\mathrm{N}_{\mathrm{Z}}<\mathrm{N}<\mathrm{N}_{\mathrm{Z}}+1\right)\end{array}\right.$

onde I, A e $\mathrm{N}_{\mathrm{Z}}$ são o potencial de ionização, a afinidade eletrônica e a soma dos números atômicos dos átomos no sistema com $\mathrm{N}$ elétrons, respectivamente.

A extensão da DFT para sistemas com um número de elétrons fracionário derivado por $\mathrm{Mermin}^{23}$ e $\mathrm{Janak}^{22}$ e sua conseqüência é discutida em detalhe por Russier ${ }^{26}$ e Perdew $^{27}$. Recentemente, Duarte e Salahub ${ }^{28}$ mostraram em detalhes estas extensões e como elas podem ajudar a aperfeiçoar o modelo de cluster para estudar adsorção de moléculas em superfícies metálicas.

\section{POTENCIAL QUÍMICO E A DEFINIÇÃO DE ELETRONEGATIVIDADE}

É uma vantagem muito grande o número de elétrons $\mathrm{N}$ e a densidade eletrônica desempenharem um papel central na Teoria do Funcional de Densidade. É muito mais difícil relacionar funções de onda de muitos elétrons com a estrutura e reatividade molecular que quantidades observáveis mais simples tais como $\rho(\mathrm{r})$ e o número de elétrons $\mathrm{N}$. A DFT permite definir também importantes conceitos químicos relacionados com a estrutura e a reatividade química de moléculas. São apresentados aqui os conceitos mais importantes que resultam da DFT e que constituem área de intensa atividade de pesquisa, ${ }^{4,29,30}$.

$\mathrm{Na}$ seção 3, ao estabelecer-se o princípio estacionário na DFT (Eq. (14)), o multiplicador de Lagrange $\mu$ foi relacionado ao potencial químico,

$\mu=\left(\frac{\partial \mathrm{E}}{\partial \mathrm{N}}\right)_{v}$

Portanto, $\mu=\mu(N, v)$ mede a tendência dos elétrons de escaparem de um sistema em equilíbrio ${ }^{31}$. Se aproximar a definição do potencial químico mostrado na equação acima pela diferença finita (veja Figura 1), chega-se a

$\mu=-\chi_{M}=-\frac{I+A}{2}$

onde I e A correspondem ao Potencial de Ionização vertical e à Afinidade Eletrônica vertical, para que seja levado em conta o fato de que o potencial externo é mantido constante (veja Eq. (30)). A Figura 1 mostra graficamente a definição de I e A, e como estas grandezas podem ser usadas para estimar a primeira e segunda derivada da energia em relação ao número de elétrons.

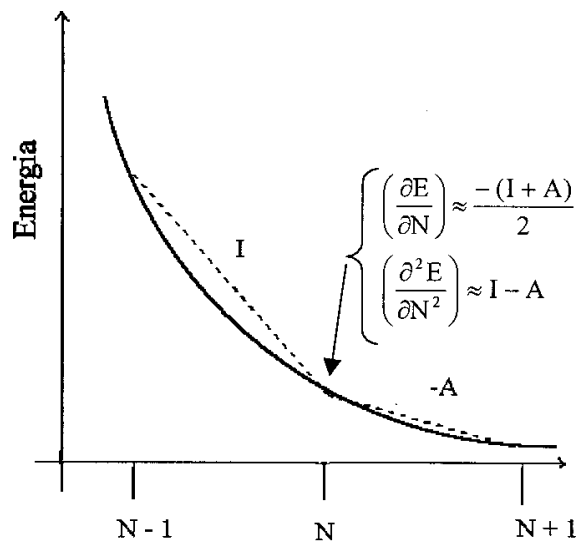

Número de Elétrons

Figura 1. Energia total do sistema em função do número de elétrons. $N$ corresponde ao número de elétrons do sistema neutro. A inclinação das linhas tracejadas correspondem ao I e A, respectivamente, dentro da aproximação da diferença finita.

Esta é a mesma definição de eletronegatividade $\left(\chi_{M}\right)$ introduzida por Mulliken ${ }^{32,33}$, em 1934. O conceito de eletronegatividade é importante na Teoria do Funcional de Densidade, uma vez que ele é o potencial químico e, por isso, desempenha um papel importante no princípio variacional, Eq. (14). Esta expressão foi identificada primeiramente por Parr et al. ${ }^{34}$.

Sanderson propôs em $1954^{35}$ o princípio da igualação da 
eletronegatividade: "quando dois ou mais átomos, inicialmente possuindo eletronegatividades diferentes, ligam-se para formar um composto, suas eletronegatividades tornam-se ajustadas a um valor intermediário dentro do composto"36, ou seja, o potencial químico em um sistema em equilíbrio é igual em toda a parte. Quando duas moléculas se aproximam, o potencial químico deve ser igualado para os dois sistemas em equilíbrio. O potencial químico (eletronegatividade) é uma propriedade de estado do sistema e pode ser calculada via DFT, Eq.(31). A transferência de elétrons entre duas moléculas é dirigida pelas diferenças do potencial químico. O formalismo DFT leva rigorosamente ao conceito de eletronegatividade e ao princípio da igualação da eletronegatividade, mesmo embora todos estes conceitos tenham sido formulados sobre uma base empírica no passado.

Vejamos o exemplo da formação do composto $\mathrm{NaF}$ a partir dos seus elementos, no estado neutro em fase gasosa ( $\mathrm{Na}$ e F). A Figura 2 mostra a formação deste composto exemplificando o princípio da igualação da eletronegatividade e o papel do potencial químico na formação da ligação química. A Figura 2a mostra a variação da energia total de cada fragmento em relação a variação da carga eletrônica e a Figura 2 b corresponde a 1a. derivada da energia total com relação a carga. Inicialmente, os dois elementos estão infinitamente separados, cada qual com a carga q igual a zero. O Flúor apresenta potencial químico em torno de 9,3 e o Sódio em torno de 3,1. Em seguida, carga eletrônica flui do flúor (com maior $\mu$ ) para o sódio (com menor $\mu)$. Em torno de $q=0,35(\mu=4,8)$ os potenciais químicos são igualados e o fluxo de carga é interrompido. A definição do potencial químico em cada fragmento desta molécula é dado pela Eq. (32). Igualado o potencial químico, o sistema se encontra em equilíbrio e o composto $\mathrm{NaF}$ é formado. Vale notar que pela análise de Mulliken, o cálculo DFT do composto NaF (distância de ligação calculada para $1,96 \AA$ ) prevê carga de 0,30 sobre o átomo de sódio e $-0,30$ sobre o átomo de flúor.

$\mu=\underbrace{\left(\frac{\partial \Delta \mathrm{E}}{\partial \mathrm{N}}\right)_{v(\mathrm{r})}}_{\text {Flúor }}=\underbrace{-\left(\frac{\partial \Delta \mathrm{E}}{\partial \mathrm{N}}\right)_{v(\mathrm{r})}}_{\text {Sódio }}$

Obviamente, esta é uma visão simplificada da formação da ligação química. Alguém poderia se perguntar como dois átomos de oxigênio (que apresentam o mesmo potencial químico) podem reagir para formar a molécula de oxigênio. A explicação é dada pelo princípio da dureza máxima sugerida por Pearson em $1987^{37}$ e que será discutida na próxima seção.

\section{O POTENCIAL QUÍMICO E SUAS DERIVADAS: DUREZA, MACIEZ E FUNÇÃO DE FUKUI}

A seguinte equação diferencial descreve um sistema eletrônico quando este muda de um estado fundamental para o outro:

$$
\mathrm{dE}=\mu \mathrm{dN}+\int \rho(\mathrm{r}) \delta v(\mathrm{r}) \mathrm{dr}
$$

deduzida a partir da generalização da teoria de perturbação de $1^{\text {a }}$ ordem para incluir mudança no número total de elétrons do sistema ${ }^{4}$.

A energia total é um funcional do número de elétrons $\mathrm{N}$ e do potencial externo $v(r)$, ou seja, $E=E[N, v]$. Logo, podese escrever a equação diferencial total da energia $E$ que relaciona a mudança do sistema de um estado fundamental para um outro:

$$
\mathrm{dE}=\left(\frac{\partial \mathrm{E}}{\partial \mathrm{N}}\right)_{v(\mathrm{r})} \mathrm{dN}+\int\left(\frac{\partial \mathrm{E}}{\partial v(\mathrm{r})}\right)_{\mathrm{N}} \delta v(\mathrm{r}) \mathrm{dr}
$$
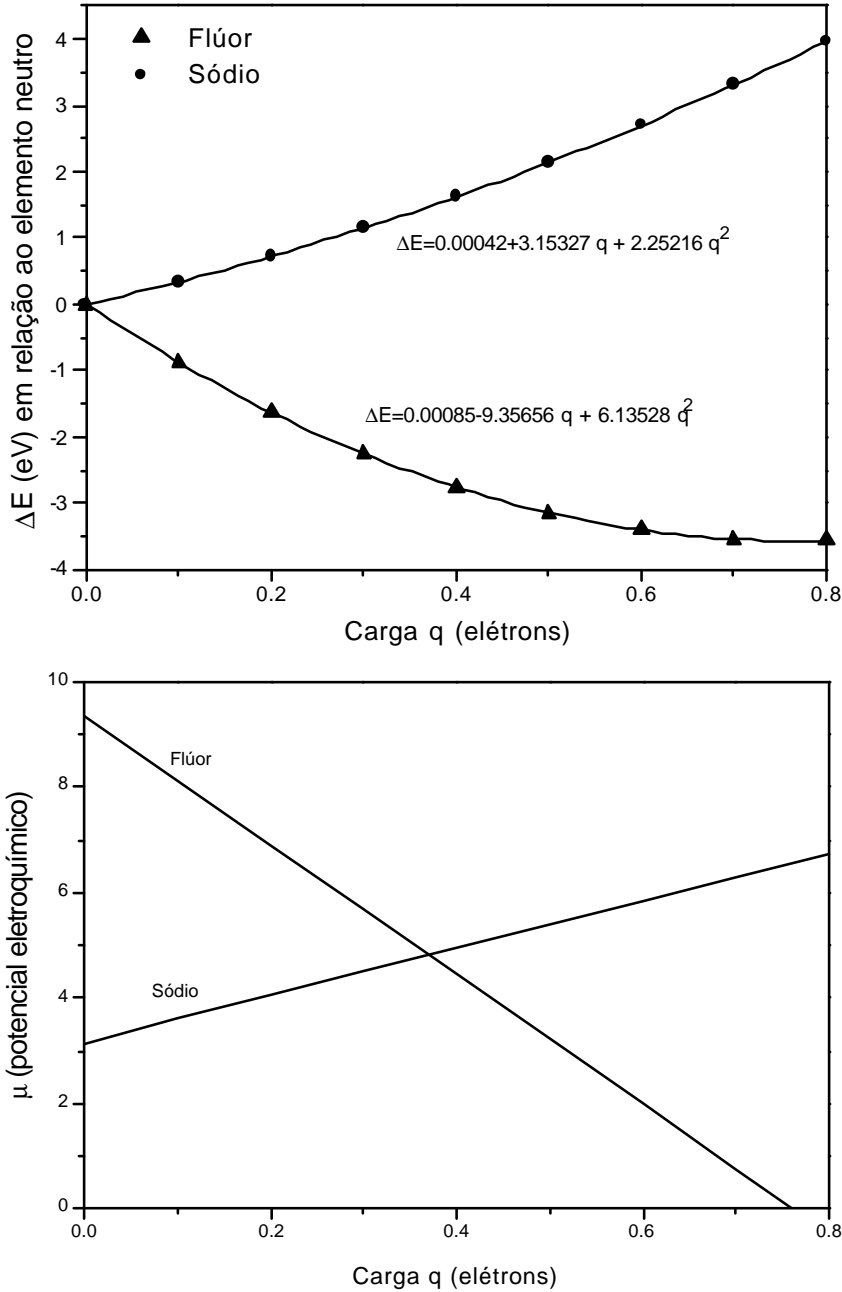

Figura 2. Variação da energia total em relação a variação da carga eletrônica na formação do NaF. A carga q representa o valor absoluto da carga fracionária (em elétrons) recebida pelo átomo fluor e doado pelo átomo sódio. A definição do potencial químico na figura é dada pela Eq. (32).

Lembrando que a partir da densidade eletrônica $\rho(\mathrm{r})$ determina-se o número de elétrons $\mathrm{N}$, podemos também escrever a expressão da energia total como um funcional da densidade eletrônica: $E=E[\rho, v]$. Logo a Eq. (34) pode ser rescrita como segue ${ }^{\mathrm{ii}}$ :

$\left.\mathrm{dE}=\int^{\prime} \frac{\partial \mathrm{E}}{\partial \rho(\mathrm{r})}\right)_{v(\mathrm{r})} \delta \rho(\mathrm{r}) \mathrm{dr}+\int\left(\frac{\partial \mathrm{E}}{\partial v(\mathrm{r})}\right)_{\rho(\mathrm{r})} \delta v(\mathrm{r}) \mathrm{dr}$

Porém, torna-se necessário mencionar que embora a Eq. (34) seja exata, a Eq. (35) é apenas uma aproximação, pois estamos assumindo que $\rho(\mathrm{r})$ e $v(\mathrm{r})$ são independentes. No entanto, os teoremas de Hohenberg-Kohn demonstram que v(r) completamente determina a densidade eletrônica do estado fundamental.

Comparando as Eqs. (33) e (34), obtem-se as seguintes igualdades:

$\mu=\left(\frac{\partial \mathrm{E}}{\partial \mathrm{N}}\right)_{v(\mathrm{r})}=$ constan $\mathrm{te}$

ii $\mathrm{O}$ diferencial de um funcional $\mathrm{F}[\mathrm{f}(\mathrm{x})]$ é dado por $\delta \mathrm{F}=\int \frac{\delta \mathrm{F}}{\delta \mathrm{f}(\mathrm{x})} \delta \mathrm{f}(\mathrm{x}) \mathrm{dx}$ 
$\left(\frac{\partial \mathrm{E}}{\partial v(\mathrm{r})}\right)_{N}=\left(\frac{\partial \mathrm{E}}{\partial v(r)}\right)_{\rho}=\rho(\mathrm{r})$

O potencial químico $\mu$, por sua vez, é um funcional do número de elétrons $\mathrm{N}$ e do potencial externo $v(\mathrm{r})$, isto é, $\mu=\mu[N, v]$. Diferenciando esta expressão temos:

$\left.\mathrm{d} \mu=\left(\frac{\partial \mu}{\partial \mathrm{N}}\right)_{v(\mathrm{r})} \mathrm{dN}+\int^{\prime} \frac{\partial \mu}{\partial v(\mathrm{r})}\right)_{\mathrm{N}} \delta v(\mathrm{r}) \mathrm{dr}$

A Eq. (38) poderia ser derivada levando-se em conta que $\mu$ é um funcional de $\rho(\mathrm{r})$ e, consequentemente, uma equação mais complexa seria obtida.

Importantes conceitos químicos surgem a partir das Eqs. (33),(34),(36) e (37):

Dureza (Hardness) $)^{38}$

$\eta=\left(\frac{\partial \mu}{\partial N}\right)_{v(r)}=\left(\frac{\partial^{2} E}{\partial N^{2}}\right)_{v(r)}$

Se utilizarmos a aproximação da diferença finita (veja Figura 1), chegamos a equação:

$\eta=\mathrm{I}-\mathrm{A}$

Esta definição é a mesma que a sugerida por Pearson em $1963^{39}$.

Recentemente, vários trabalhos foram publicados mostrando a aplicação destes conceitos para a compreensão do processo de isomerização em compostos como peróxido de hidrogêio, tioperóxido de hidrogênio e persulfeto de hidrogênio. ToroLabbé et al. $^{40}$ mostraram como o potencial químico e a dureza variam com a ângulo de torção da molécula. Embora eles tenham usado em seus cálculos a aproximação da diferença finita, ou seja, $\eta=1 / 2\left(E_{L}-E_{H}\right)$, onde $E_{L}$ e $E_{H}$ correspondem a energia do LUMO e HOMO, respectivamente, os resultados se comportam como o esperado. A dureza apresenta um máximo onde se encontra os mínimos de energia na superfície de energia potencial. O princípio da dureza máxima foi também exemplificada no estudo do rearranjo intramolecular de uma série de moléculas tais como $\mathrm{HCN}, \mathrm{HClO}, \mathrm{HONS}, \mathrm{H}_{2} \mathrm{SO}, \mathrm{F}_{2} \mathrm{~S}_{2}$, $\mathrm{H}_{3} \mathrm{PO}, \mathrm{CH}_{2} \mathrm{SH}_{2}{ }^{41}$. Em todos estes sistemas a dureza é máxima nos pontos correspondentes aos mínimos na superfície de energia potencial.

Maciez (Softness), que é o inverso da Dureza ${ }^{42}$ :

$S=\left(\frac{\partial N}{\partial \mu}\right)_{v(r)}=\frac{1}{I-A}$

Estes conceitos propostos inicialmente sobre uma base empírica estão, a partir do formalismo DFT, precisamente definidos.

$\mathrm{O}$ princípio enunciado por Pearson ${ }^{39}$ : "ácidos macios preferem bases macias, ácidos duros preferem bases duras", também chamado de princípio HSAB (do inglês: Hard Soft Acid Base principle) também está contemplado dentro da DFT. Porém não é uma tarefa fácil estabelecer teoricamente este princípio. Recentemente, Parr e co-autores ${ }^{43}$, forneceram duas demonstrações deste princípio com a restrição de que os dois reagentes tenham a mesma eletronegatividade, ao invés do potencial externo constante. A caracterização do tautomerismo ketoenol dos derivados do acetil foram analisados recentemente em termos do potencial químico e dureza ${ }^{44}$. O princípio HSAB foi invocado mostrando o bom acordo com os dados termodinâmicos. De acordo com o princípio $\mathrm{HSAB}$, a forma keto é favorecida em relação a forma enol.

Índice de Reatividade de Fukui (ou Função de Fukui):

$\mathrm{f}(\mathrm{r})=\left[\frac{\partial \mu}{\partial v(\mathrm{r})}\right]_{\mathrm{N}}=\left[\frac{\partial \rho(\mathrm{r})}{\partial \mathrm{N}}\right]_{v}$

Este índice de reatividade mede como uma perturbação externa afeta o potencial químico de um sistema. A função de Fukui, f(r), envolve a densidade eletrônica do átomo ou molécula na sua região de valência. Na realidade, a função de Fukui pode ser usada para medir a reatividade em relação a um ataque nucleofílico, eletrofílico ou mesmo frente a um reagente radicalar ${ }^{45}$. Três definições numéricas foram propostas para as funções de Fukui por Yang e Parr ${ }^{46}$. Estas três definições são devidas ao fato de que a Eq. 42 apresenta descontinuidade para $\mathrm{N}$ igual a um número inteiro.

$\mathrm{f}^{+}(\mathrm{r})=\left(\frac{\partial \rho(\mathrm{r})}{\partial \mathrm{N}}\right)_{v}^{+}:$governa ataque nucleofílico

$\mathrm{f}^{-}(\mathrm{r})=\left(\frac{\partial \rho(\mathrm{r})}{\partial \mathrm{N}}\right)_{0}^{-}:$governa ataque eletrofílico

$\mathrm{f}^{0}(\mathrm{r})=\left(\frac{\partial \rho(\mathrm{r})}{\partial \mathrm{N}}\right)_{v}^{0}:$ governa ataque de radical

Em outras palavras, se usarmos a aproximação da diferença finita nas definições apresentadas nas Eqs 43 e 44, tem-se que:

$\mathrm{f}^{-}(\mathrm{r})=\rho_{\mathrm{N}=\mathrm{N}_{0}}(\mathrm{r})-\rho_{\mathrm{N}=\mathrm{N}_{0}-1}(\mathrm{r})=\rho_{\mathrm{HOMO}}(\mathrm{r})$

$\mathrm{f}^{+}(\mathrm{r})=\rho_{\mathrm{N}=\mathrm{N}_{0}+1}(\mathrm{r})-\rho_{\mathrm{N}=\mathrm{N}_{0}}(\mathrm{r})=\rho_{\mathrm{LUMO}}(\mathrm{r})$

A terceira função, $\mathrm{f}^{0}(\mathrm{r})$, é estimada como uma média das funções $\mathrm{f}^{+}(\mathrm{r})$ e $\mathrm{f}^{-}(\mathrm{r})$. Em contraste com os conceitos de dureza e maciez que são quantidades globais, a função de Fukui é uma quantidade local e reflete as propriedades dos diferentes sítios dentro de uma molécula. Usando da aproximação dada pelas Eqs 46 e 47, a Figura 3 mostra os orbitais HOMO e LUMO do cloreto de t-butila indicando onde um ataque nucleofílico ou eletrofílico pode acontecer.

A energia total de um sistema pode ser expandida em termos destas grandezas, como se segue abaixo ${ }^{47}$ :

$E[N, v]=N \mu-\frac{1}{2} N^{2} \eta+\int v(r)[\rho(r)-N f(r)] d r+\cdots$

Outras grandezas importantes para a química e física surgem a partir da DFT tais como os conceitos de maciez local (Eq. 49) e sua inversa, chamada de dureza local.

$s(r)=\left[\frac{\partial \rho(r)}{\partial \mu}\right]_{v}=f(r) S$

A Eq. 49 mostra que a maciez pode ser determinada em diferentes regiões da molécula e, deste modo, avaliando a reatividade nos diferentes sítios de um sistema molecular.

Os conceitos relacionados ao potencial químico e suas 


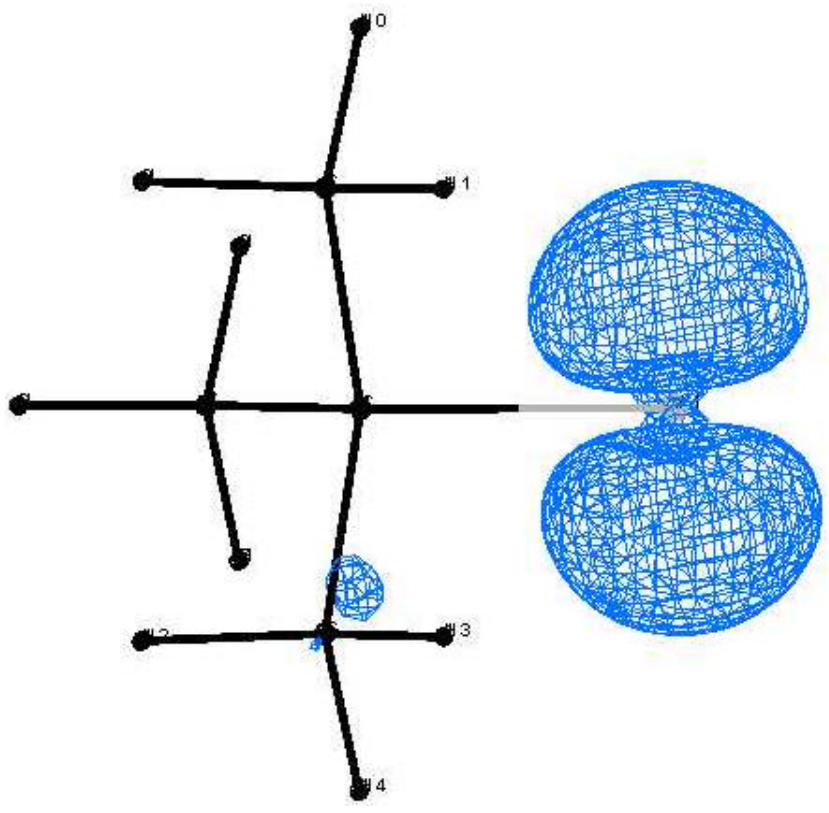

HOMO

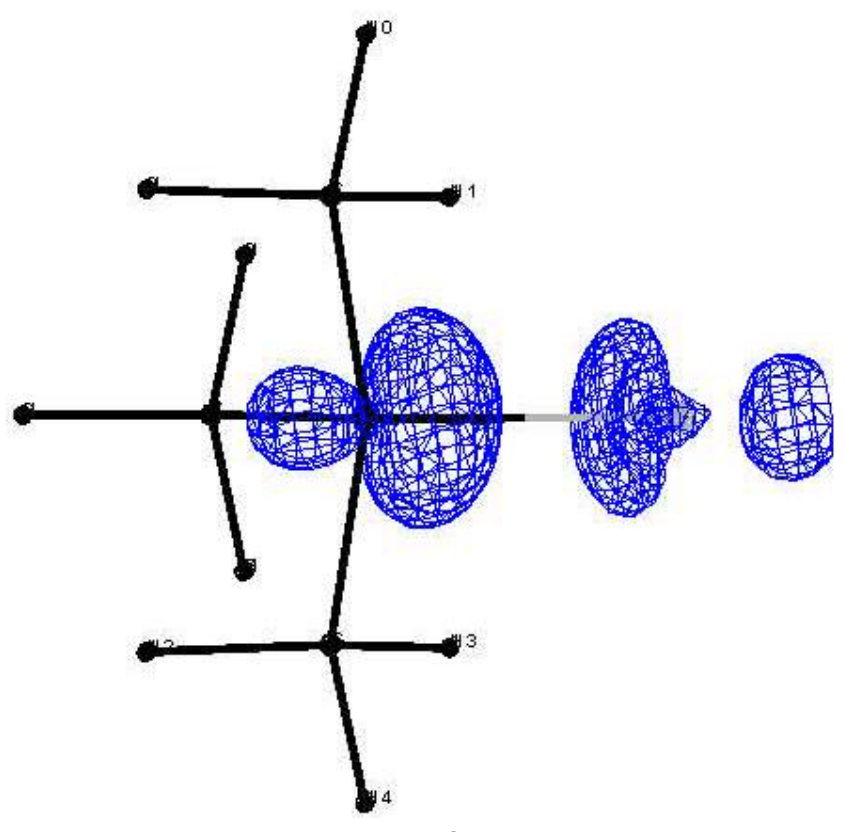

LUMO

Figura 3. As superfícies referem-se aos contornos dos orbitais de fronteira do Cloreto de t-butila. (0,02 au).

derivadas foram discutidas em detalhes na caracterização de reações químicas por Toro-Labbé ${ }^{48}$. Estes conceitos foram aplicados no estudo da transferência de prótons em complexos do tipo CHX$\mathrm{XH} \ldots \mathrm{CHX}-\mathrm{XH}(\mathrm{X}=\mathrm{O}, \mathrm{S})^{49}$ e para estimar energias de ligação de complexos ligados por ligações de hidrogênio ${ }^{50}$. O sucesso na aplicação dos conceitos de potencial químico, dureza e maciez para se caracterizar reações químicas é notório, apesar de na grande maioria dos trabalhos utilizarem aproximações da diferença finita para avaliar estas propriedades. Em relação as propriedades locais tais como a dureza e maciez locais além da função de Fukui ainda são muito incipientes, embora sejam de óbvio interesse.

\section{CONSIDERAÇÕES FINAIS}

Sabemos há muito tempo que a química teórica já chegou a um ponto em seu desenvolvimento no qual cálculos teóricos tem, em muitas situações, o status de experimento, dada a sua precisão. A química teórica tem sido utilizada intensamente no auxílio da interpretação de resultados experimentais, na avaliação da viabilidade de determinadas rotas sintéticas, na compreensão de fenômenos nos quais as técnicas experimentais não podem ainda ser diretamente utilizadas, no estudo de caminhos de reações, de barreiras de reação, da influência de ligantes em compostos de coordenação, na espectroscopia do infra-vermelho (IR) e no VIS-UV, etc ${ }^{51}$. No entanto, sabemos que ainda é um grande desafio interpretar e transmitir as informações, geradas pelos cálculos teóricos, numa linguagem capaz de ser facilmente compreendida por um químico experimental. Em relação a este último ponto, a Teoria do Funcional de Densidade apresenta uma grande vantagem. A descrição da energia eletrônica e das propriedades moleculares a partir de uma quantidade observável, a densidade eletrônica, permite uma comunicação mais objetiva entre teóricos e experimentais. Vale ressaltar que a Teoria do Funcional de Densidade, em seu formalismo, levanta o véu sobre importantes aspectos da química que ainda eram vistos à luz do empirismo. Conceitos como potencial químico, $\mu$, maciez, dureza e índice de reatividade formalmente definidos a partir da DFT abrem uma nova perspectiva na compreensão dos fenômenos químicos. Tais quantidades podem ser combinadas em uma equação fundamental:

$d \mu=\eta d N+\int f(r) d v(r) d r$

O potencial químico e seus derivados são atualmente objeto de intensa pesquisa (veja, por exemplo, as referências 29,30,52,53) A DFT fornece uma descrição da estrutura dos átomos, moléculas, clusters, superfícies e sólidos. De acordo com Kohn ${ }^{\mathrm{iii}}$ et $\mathrm{al}^{29}$, e com eles estamos em pleno acordo, DFT é uma linguagem conveniente e universal para a teoria de estrutura eletrônica, a qual ajuda substancialmente a unificar a química orgânica, a inorgânica, química de superfície e a ciência dos materiais. Podemos ainda acrescentar que as dificuldades em implementar o cálculo de índices de reatividade (maciez, dureza, funções de Fukui, etc.) de forma conveniente a um custo computacional acessível estão sendo devidamente tratados. Deste modo, acreditamos que a DFT ocupará um espaço ainda maior, tornando-se também uma ferramenta de grande utilidade no estudo de sistemas bioquímicos, interações droga-aceptor e mecanismos de atividade biológica.

\section{AGRADECIMENTOS}

Gostaria de agradecer igualmente aos Profs. Eucler B. Paniago e Wagner B. De Almeida pelas contribuições e sugestões a este trabalho. Agradecemos também ao Centro Nacional de Processamento de Alto Desempenho - CENAPAD-MG/CO pelo acesso aos seus recursos computacionais e ao Conselho Nacional de Desenvolvimento Científico - CNPq, pelo apoio financeiro.

\section{REFERÊNCIAS}

1. Drude, P.; Annalen der Physik 1900, 1, 566. Drude, P.; Annalen der Physik 1900, 3, 369.

2. Fermi, E.; Rend. Accad. Lincei 1927, 6, 602. Thomas, L. H.; Proc. Camb. Phil. Soc. 1927, 23, 542.

3. Dirac, P. A. M.; Proc. Camb. Phil. Soc. 1930, 26, 376.

4. Parr, R. G.; Yang, W.; Density Functional Theory of Atoms and Molecules, Oxford University Press, Oxford, 1989.

5. Dreizler, R, M,; Gross, E. K. U.; Density Functional Theory. An Approach to the Quantum Many-Body Problem, Springer-Verlag, Berlin, 1990.

\footnotetext{
iii Agraciado, juntamento com o J. Pople, com o prêmio Nobel de química, 1998.
} 
6. Hohenberg, P.; Kohn, W.; Phys. Rev. 1964, 136, B864.

7. Kohn, W.; Sham, L. J.; Phys. Rev. 1965, 140, A1133.

8. Morgon, N. H.; Custódio, R.; Quim. Nova 1995, 18, 44.

9. Gilbert, T. L.; Phys. Rev. B 1975, 12, 2111.

10. Levy, M.; Proc. Natl. Acad. Sci. USA 1979, 76, 6062. Levy, M.; Phys. Rev. A. 1982, 26, 1200. Levy, M; Perdew, J. P.; Phys. Rev. A 1985, 32, 2010.

11. Baerends, E. J.; Gritsenko, O. V.; J. Phys. Chem. A 1997, 30, 5383 .

12. Parr, R. G.; Yang, W., Annu. Rev. Phys. Chem. 1995, 46, 701.

13. Perdew, J. P.; Burke, K.; Intern. J. Quantum Chem. 1996, 57, 309.

14. Vosko, S. H.; Wilk, L.; Nusair, M.; Can. J. Phys. 1980, $58,1200$.

15. Slater J. C.; Phys. Rev. 1951, 81, 385.

16. Becke, A. D.; J. Chem. Phys. 1986, 84, 4524.

17. Perdew, J. P.; Phys. Rev. Lett. 1985, 55, 1665.

18. Perdew, J. P.; Yue, W.; Phys. Rev. B 1986, 33, 8800.

19. Lee, C.; Yang, W.; Parr, R. G.; Phys. Rev. B 1988, 37,785 .

20. Becke, A. D.; Phys. Rev. A 1988, 38, 3098.

21. Perdew, J. P.; Phys. Rev. B 1986, 33, 8822. Ibid. 1986, 34, 7406.

22. Janak, J. F.; Phys. Rev. B 1978, 18, 7165.

23. Mermin, N. D.; Phys. Rev. 1965, 137, A1441.

24. Perdew, J. P.; Parr, R. G.; Levy, M.; Balduz, J. L. Jr.; Phys. Rev. Lett. 1982, 49, 1691.

25. Perdew, J. P.; Zunger, A.; Phys. Rev. B 1981, 23, 5048.

26. Russier, V.; Phys. Rev. B 1992, 45, 8894.

27. Perdew, J. P., In.: Density Functional Methods in Physics, R. M. Dreizler and J. da Providência, Eds; NATO ASI Series-B Bol. 123, 1985.

28. Duarte, H. A.; Salahub, D. R.; J. Chem. Phys. 1998, 108, 743.

29. Kohn, W; Becke, A. D.; Parr, R. G.; J. Phys. Chem. 1996, 100, 12974.

30. Chermette, H; J. Comp. Chem. 1999, 20, 129.

31. Parr, R. G.; Donnelly, R. A.; Levy, M. and Palke, W. E.; J. Chem. Phys. 1978, 68, 3801.

32. Mulliken, R. S.; J. Chem. Phys. 1934, 2, 782.

33. Gyftopoulos, E. P.; Hatsopoulos, G. N.; Proc. Natl. Acad. Sci. USA 1965, 60, 786.

34. Parr, R. G.; Donnelly, R. A.; Levy, M.; Palke, W. E.; J. Chem. Phys. 1978, 68, 3801.
35. Sanderson, R. T.; J. Chem. Educ. 1952, 29, 539. Ibid. 1954, 31, 238.

36. Sanderson, R. T.; Chemical Bounds and Bound Energy; Academic: New York, 1971.

37. Pearson, R. G.; J. Chem. Educ. 1987, 64, 561.

38. Parr, R. G.; Pearson, R. G.; J. Am. Chem. Soc. 1983, $105,105$.

39. Pearson, R. G.; J. Am. Chem. Soc. 1963, 85, 3533.

40. Gutiérrez-Oliva, S.; Letelier, J. R.; Toro-Labbé, A.; Mol. Phys. 1999, 96, 61.

41. Solá, M.; Toro-Labbé, A.; J. Phys. Chem. A 1999, 103, 8847

42. Yang, W.; Parr, R. G.; Proc. Natl. Acad. Sci. USA 1985, 82,6723

43. Chattaraj, P. K.; Lee, H.; Parr, R. G.; J. Am. Chem. Soc. 1991, 113, 1855.

44. Péreza, P.; Toro-Labbé, A.; J. Phys. Chem. A 2000, 104, 1557.

45. Lee, C., Yang, W., Parr, R. G., J. Mol. Structure (Theochem) 1987, 163, 305.

46. Perdew, J. P.; Parr, R. G. ; Levy, M.; Balduz, J. L.; Phys. Rev. Lett. 1982, 49, 1691.

47. Liu, S; Parr, R. G.; J. Chem. Phys. 1997, 106, 5578, 5586.

48. Toro-Labbé, A.; J. Phys. Chem. A. 1999, 103, 4398.

49. Jaque, P.; Toro-Labbé, A.; J. Phys. Chem. A 2000, 104, 995.

50. Gutiérrez-Oliva, S.; Jaque, P.; Toro-Labbé, A.; J. Phys. Chem. A 2000, 104, 8955.

51. Veja, por exemplo, alguns dos trabalhos desenvolvidos em nosso laboratório: Haslett, T. L., Fedrigo, S.; Bosnick, K.A.; Bier, K. D.; Moskovits, M.; Duarte, H. A.; Salahub, D.; J. Am. Chem. Soc. 2000, 122, 6039. Ladeira, A. C. Q.; Alves, M. C. M.; Duarte, H. A.; Ciminelli, V. S. T.; Geochim. Cosmochim. Acta 2001, 65, 1211. Oliveira, M. A.; Pernaut, J-M.; Duarte, H. A.; De Almeida, W. B.; J. Phys. Chem. A 2000, 104, 8256. Duarte, H. A.; Salahub, D. R.; Haslett, T.; Moskovits, M.; Inorg. Chem. 1999, 38, 3895. Duarte, H. A.; Salahub, D. R.; Top. Catal. 1999, 9, 123. Duarte, H. A.; Carvalho, S.; Paniago, E. B.; De Almeida; W. B.; J. Inorg. Biochem. 1998, 72, 71.

52. De Proft, F.; Liu S.; Parr, R. G.; J. Chem. Phys. 1997, 107, 3000.

53. Mineva, T.; Russo, N.; Sicilia, E.; Toscano, M.; J. Chem. Soc. Far. Trans. 1997, 93, 3309. 